\title{
A single parameter determines mesophases transitions in swollen Liquid Crystals
}

\section{Supplementary Information}

Natacha Kinadjian, Frédéric Nallet, Isabelle Ly, Ahmed Bentaleb, Rénal Backov and Eric

$$
\text { Prouzet }{ }^{*}
$$



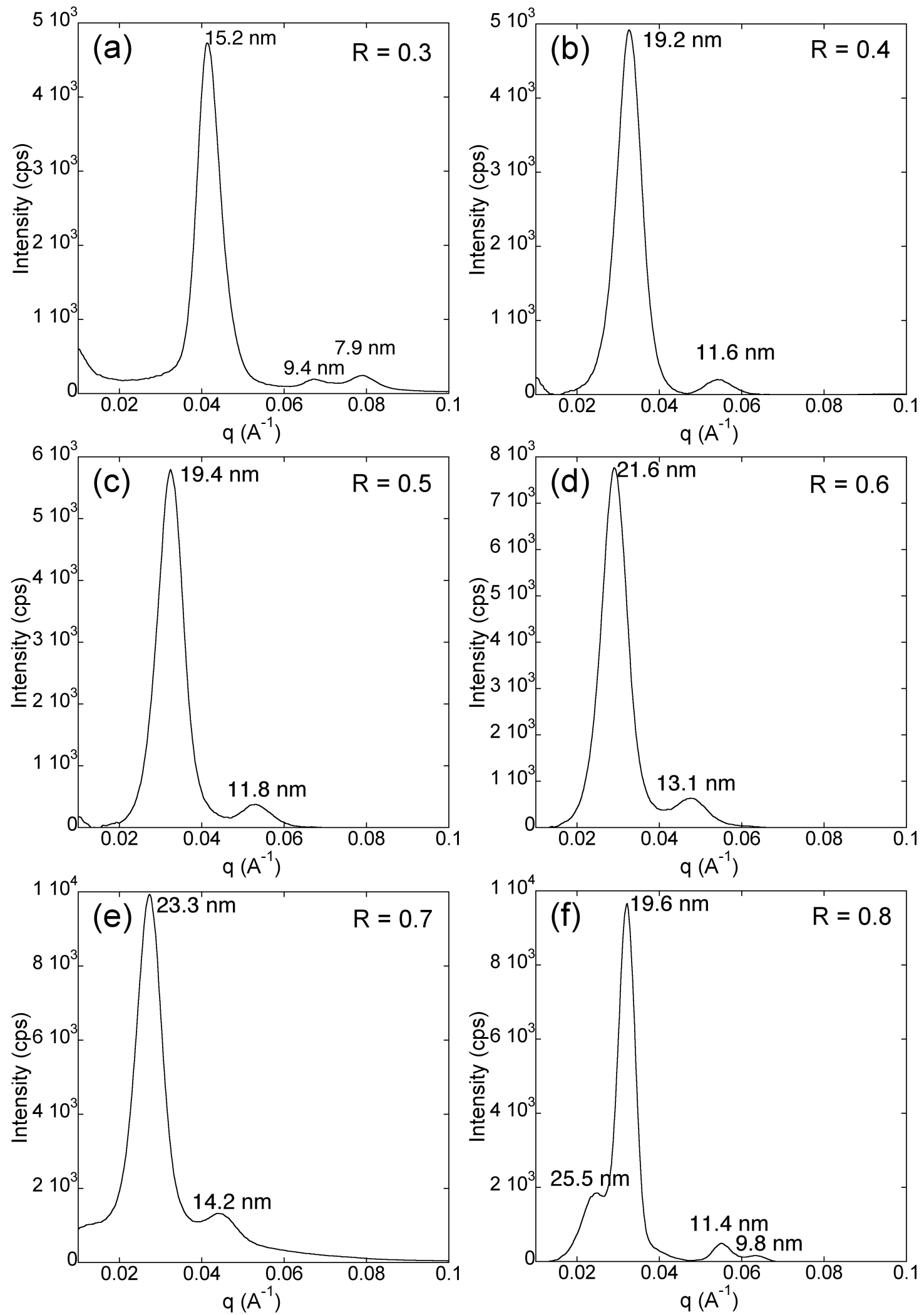

Figure S1 A: SAXS patterns evolution depending on the 1-pentanol over surfactant molar ratio $(\mathrm{R})$ varying from 0.3 to 0.8 and $[\mathrm{AP}]=0.1 \mathrm{M}(1.0 \mathrm{~g} \mathrm{CTAB}, 0.046 \mathrm{~g} \mathrm{AP}, 2.00 \mathrm{~g}$ water $(\mathrm{pH}$ 2), $3.06 \mathrm{~g}$ cyclohexane). 

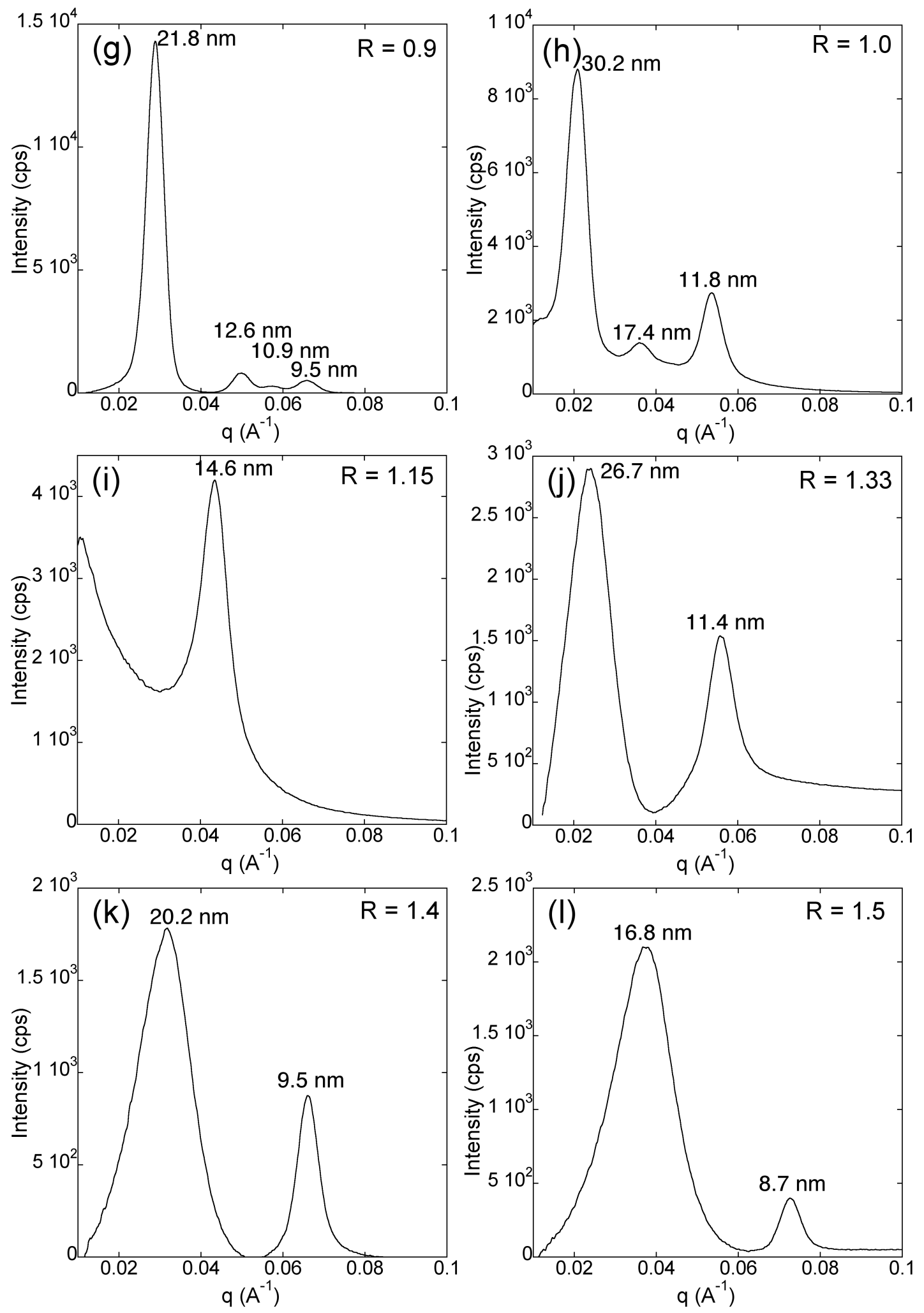

Figure S1 B: SAXS patterns evolution depending on the 1-pentanol over surfactant molar ratio $(\mathrm{R})$ varying from 0.9 to 0.1 .5 , and $[\mathrm{AP}]=0.1 \mathrm{M}(1.0 \mathrm{~g} \mathrm{CTAB}, 0.046 \mathrm{~g} \mathrm{AP}, 2.00 \mathrm{~g}$ water (pH 2), 3.06g cyclohexane). 

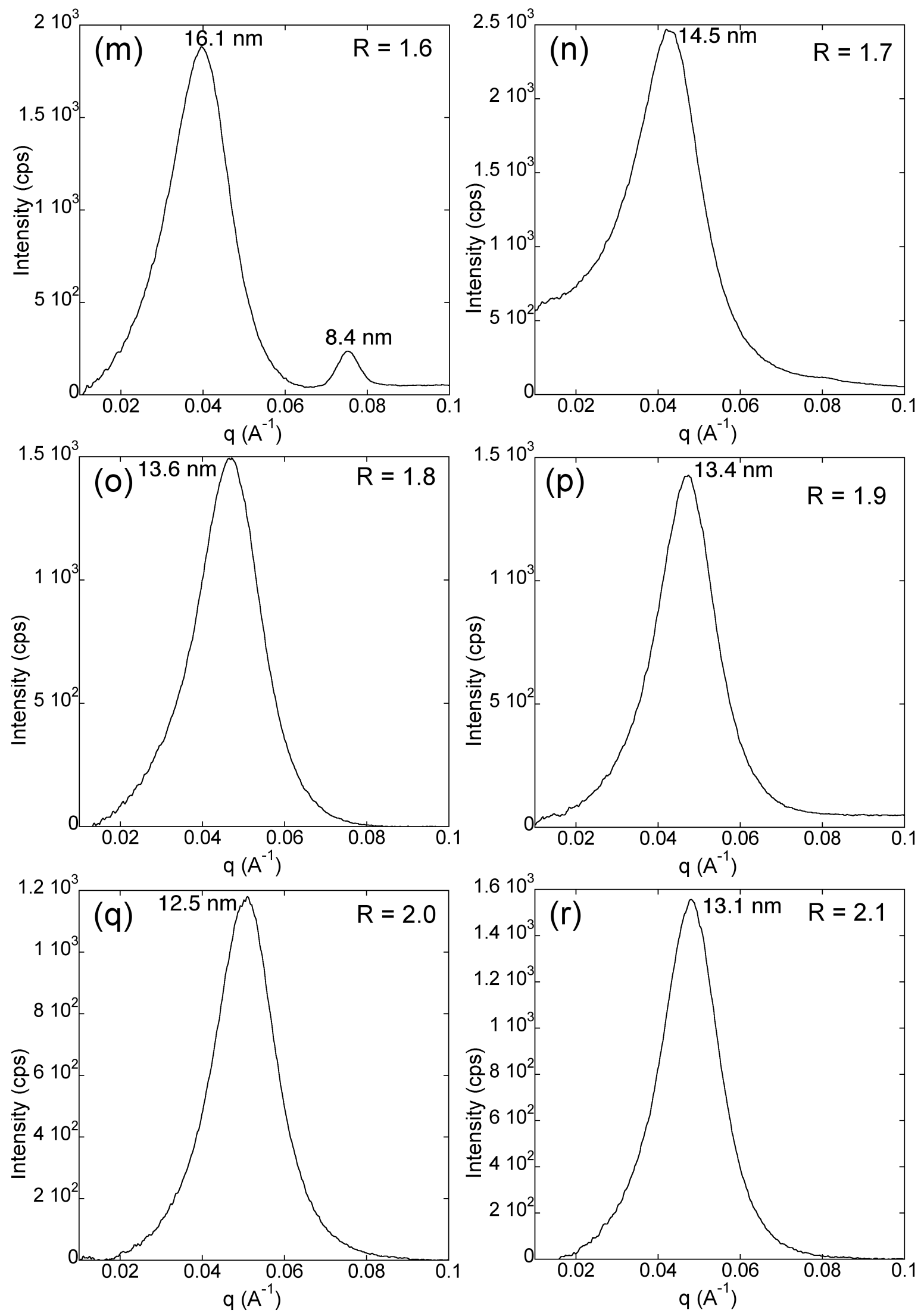

Figure S1 C: SAXS patterns evolution depending on the 1-pentanol over surfactant molar ratio $(\mathrm{R})$ varying from 1.6 to 2.1 , and $[\mathrm{AP}]=0.1 \mathrm{M}(1.0 \mathrm{~g} \mathrm{CTAB}, 0.046 \mathrm{~g} \mathrm{AP}, 2.00 \mathrm{~g}$ water $(\mathrm{pH}$ 2), $3.06 \mathrm{~g}$ cyclohexane). 


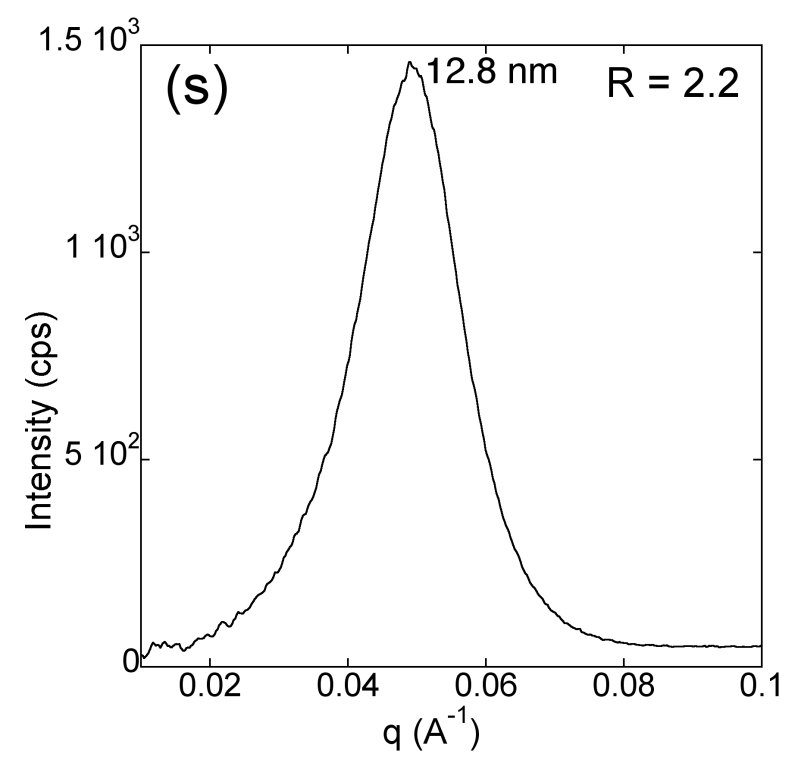

Figure S1 D: SAXS patterns evolution depending on the 1-pentanol over surfactant molar ratio $(\mathrm{R})$ equal to 2.2 , and $[\mathrm{AP}]=0.1 \mathrm{M}(1.0 \mathrm{~g} \mathrm{CTAB}, 0.046 \mathrm{~g} \mathrm{AP}, 2.00 \mathrm{~g}$ water $(\mathrm{pH} 2), 3.06 \mathrm{~g}$ cyclohexane). 

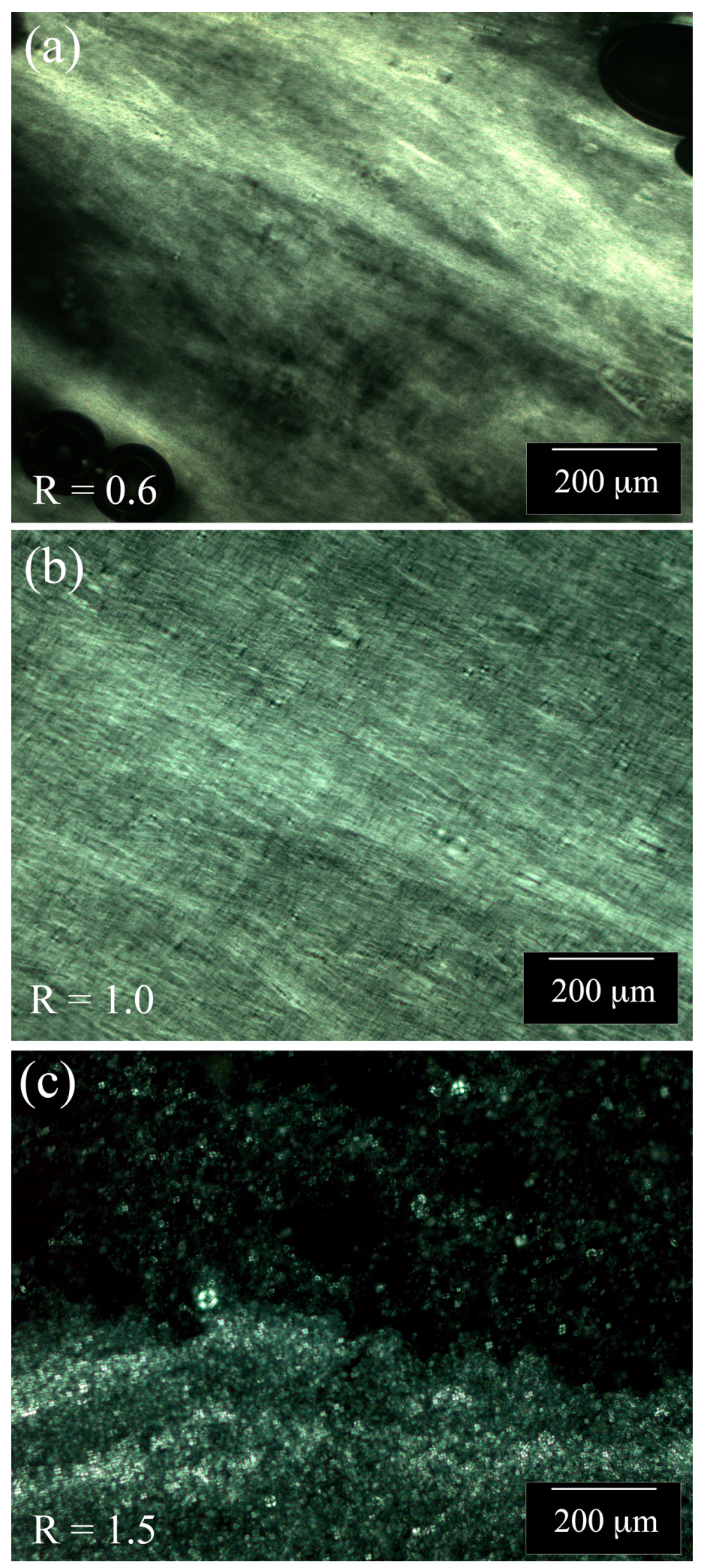

Figure S2: Optical microscopy under cross-polarized light of the GLCs made with [AP]= $0.15 \mathrm{M}$ : a) $\mathrm{R}=0.6, \mathrm{~b}) \mathrm{R}=1.0$, c) $\mathrm{R}=1.5$. 

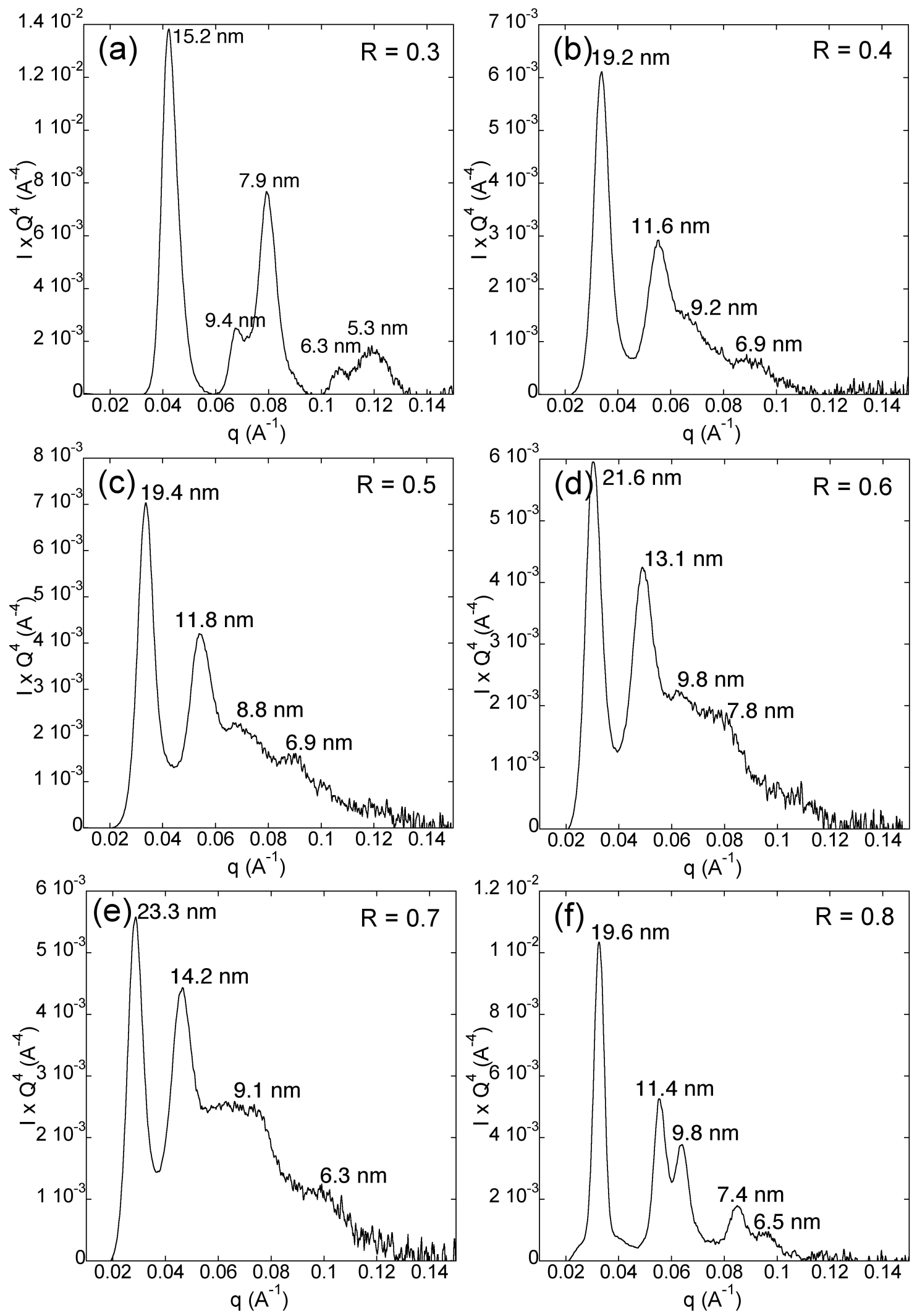

Figure S3 A: Porod representation of SAXS patterns evolution depending on the 1-pentanol over surfactant molar ratio $(\mathrm{R})$ varying from 0.3 to 0.8 , and $[\mathrm{AP}]=0.1 \mathrm{M}(1.0 \mathrm{~g} \mathrm{CTAB}, 0.046 \mathrm{~g}$ AP, 2.00g water ( $\mathrm{pH} 2), 3.06 \mathrm{~g}$ cyclohexane). 

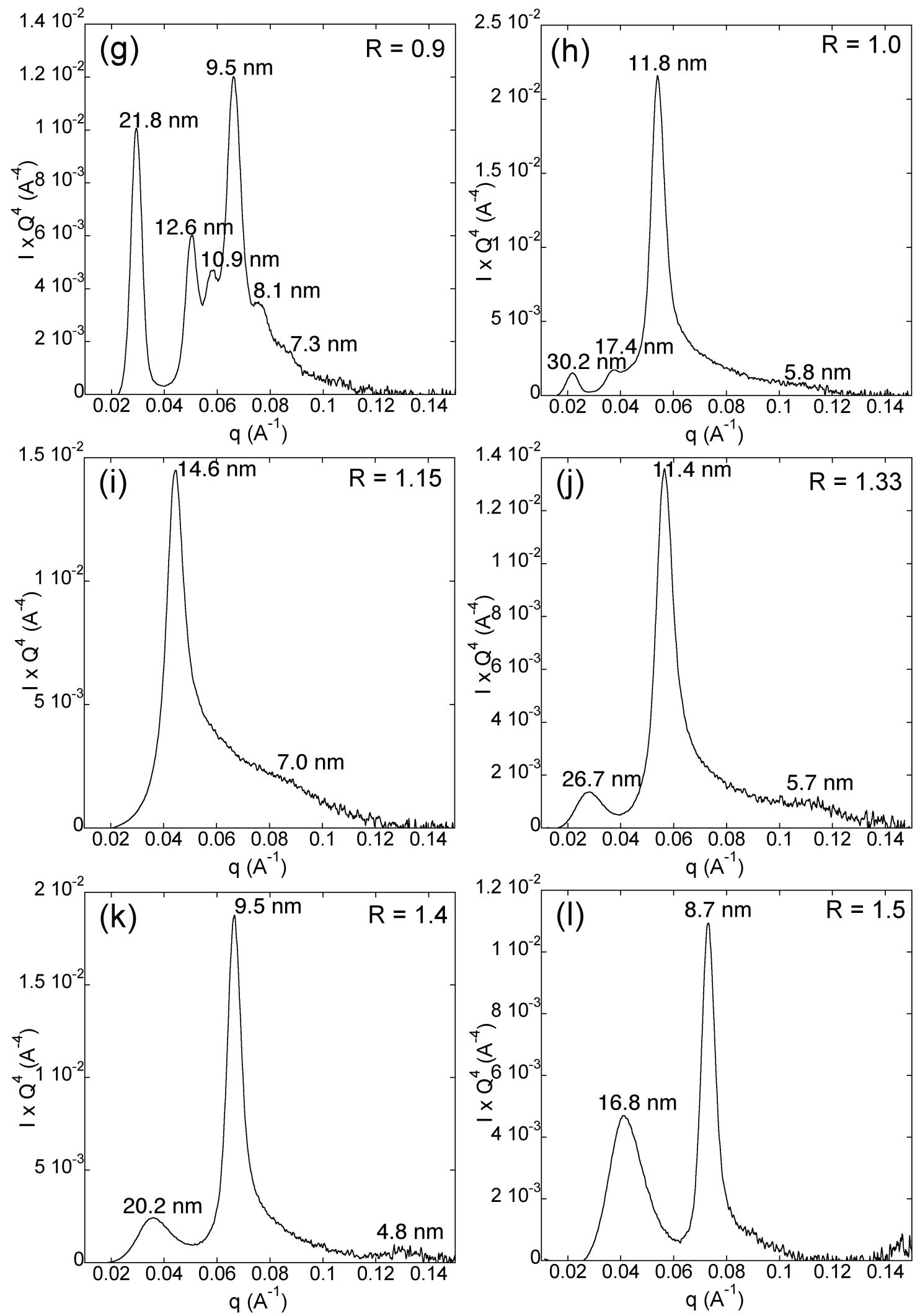

Figure S3 B: Porod representation of SAXS patterns evolution depending on the 1-pentanol over surfactant molar ratio $(\mathrm{R})$ varying from 0.9 to 1.6 , and $[\mathrm{AP}]=0.1 \mathrm{M}(1.0 \mathrm{~g}$ CTAB, $0.046 \mathrm{~g}$ AP, 2.00g water ( $\mathrm{pH} 2), 3.06 \mathrm{~g}$ cyclohexane). 

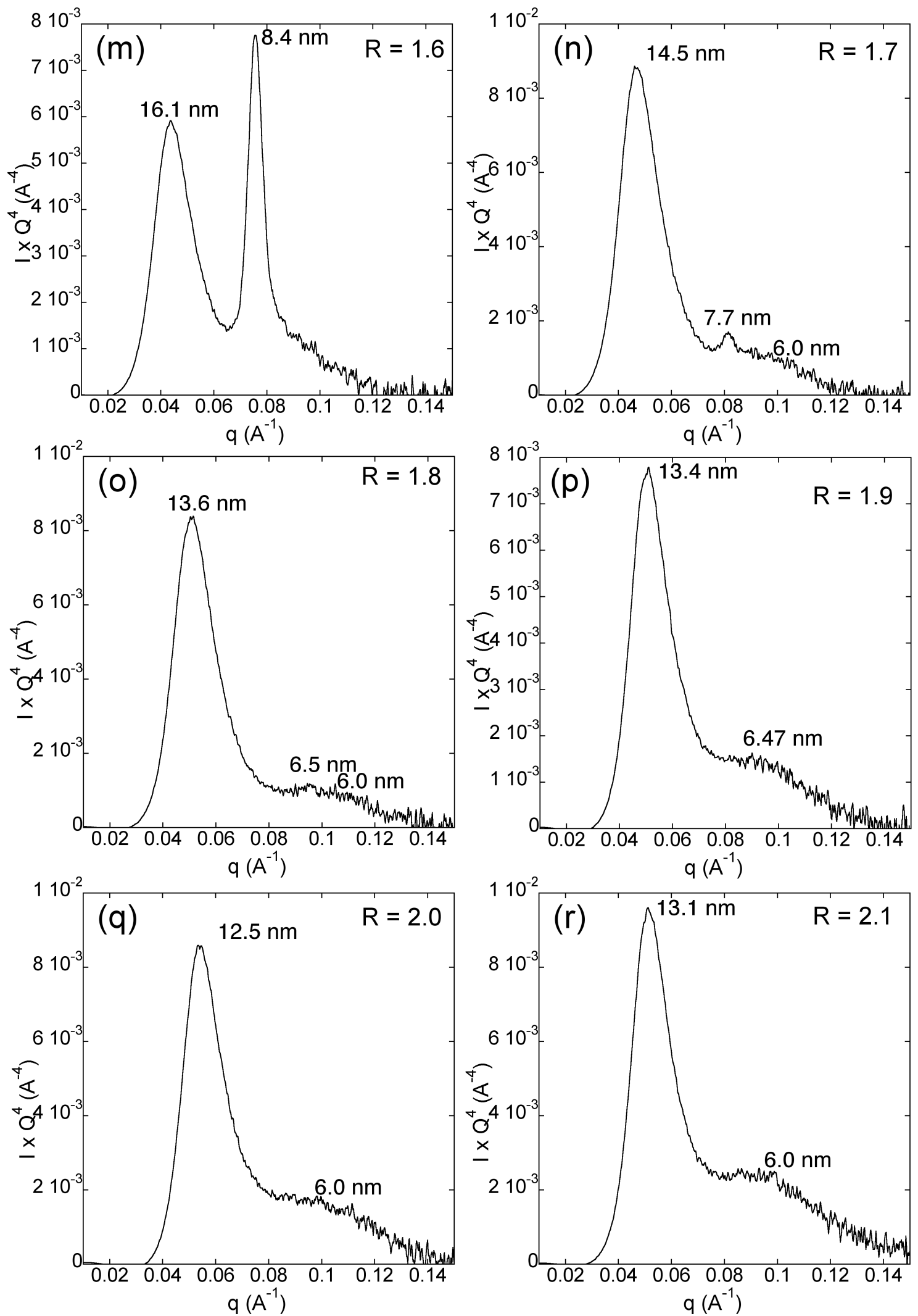

Figure S3 C: Porod representation of SAXS patterns evolution depending on the 1-pentanol over surfactant molar ratio $(\mathrm{R})$ varying from 1.7 to 2.2 , and $[\mathrm{AP}]=0.1 \mathrm{M}(1.0 \mathrm{~g} \mathrm{CTAB}, 0.046 \mathrm{~g}$ AP, $2.00 \mathrm{~g}$ water ( $\mathrm{pH} 2), 3.06 \mathrm{~g}$ cyclohexane). 


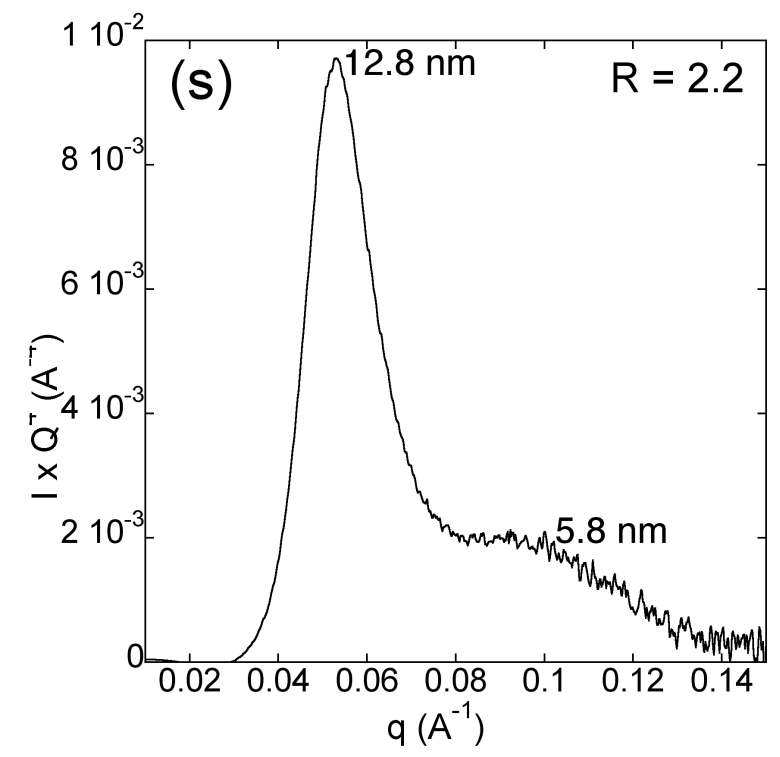

Figure S3 D: Porod representation of SAXS patterns evolution depending on the 1-pentanol over surfactant molar ratio $(\mathrm{R})$ equal to 2.2 , and $[\mathrm{AP}]=0.1 \mathrm{M}(1.0 \mathrm{~g} \mathrm{CTAB}, 0.046 \mathrm{~g} \mathrm{AP}, 2.00 \mathrm{~g}$ water (pH 2), $3.06 \mathrm{~g}$ cyclohexane). 

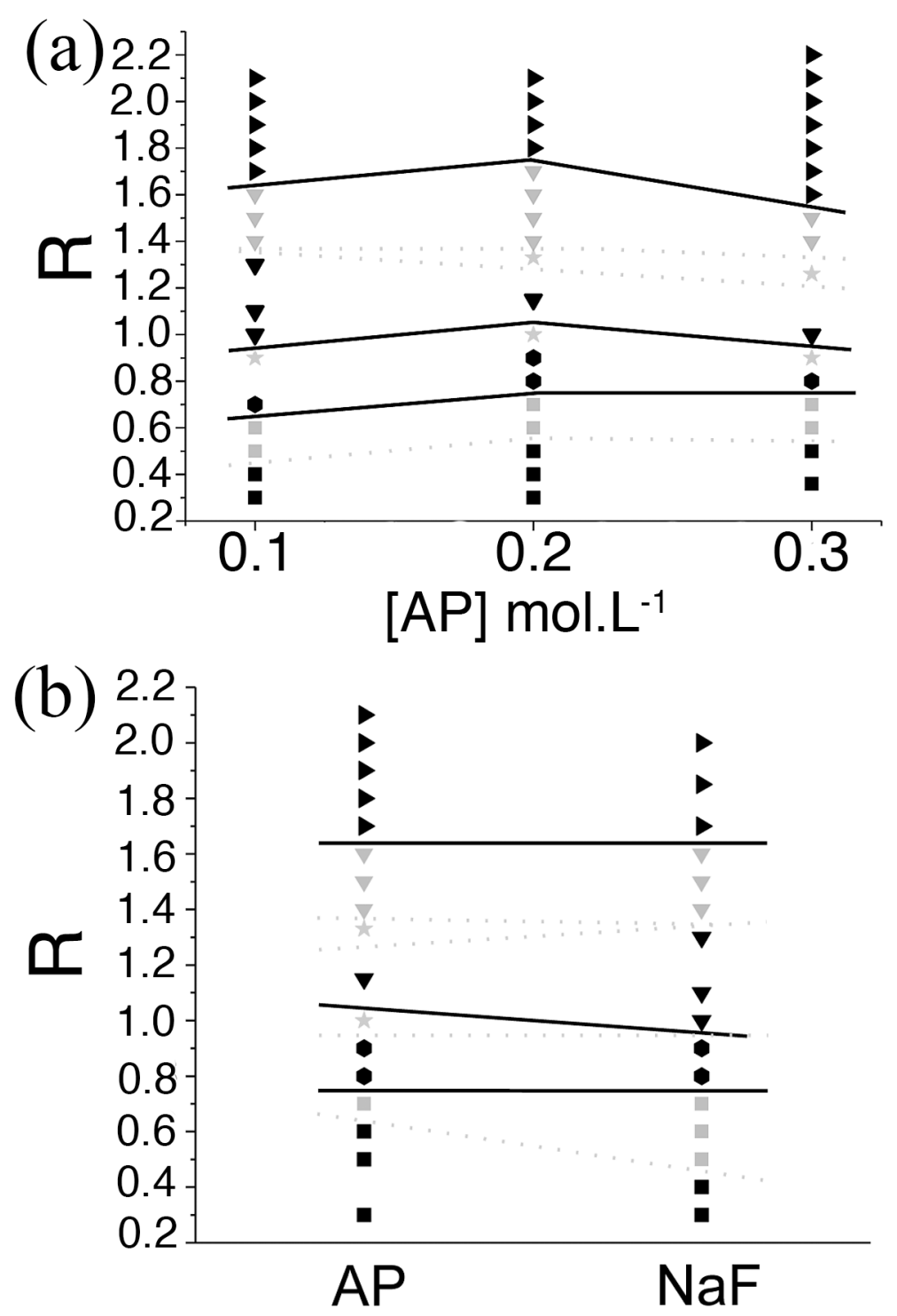

Figure S4: Evolution of the SLC mesophase structure with R as a function of (a) ammonium persulfate (AP), and (b) comparison between $0.1 \mathrm{M}$ AP and $0.2 \mathrm{M} \mathrm{NaF}$ : cubic phase, mixed cubic/hexagonal phase, $\bullet$ hexagonal phase, $\star$ “intermediary" phase, $\boldsymbol{\nabla}$ lamellar phase, $\nabla$ mixed lamellar/sponge phase, and $\boldsymbol{~ s p o n g e ~ p h a s e . ~}$ 
Table S1: d-spacing values deduced from the Porod analysis of SLC mesophases (Figure S3) prepared with different values of $\mathrm{R}$ ( $\mathrm{R}$ : co-surfactant over surfactant molar ratio). $[\mathrm{AP}]=$ $0.1 \mathrm{M}, 1.0 \mathrm{~g}$ CTAB, $0.046 \mathrm{~g}$ AP, $2.00 \mathrm{~g}$ water $(\mathrm{pH} 2), 3.06 \mathrm{~g}$ cyclohexane.

\begin{tabular}{c|lllll}
\hline $\mathrm{R}$ & \multicolumn{5}{|c}{$\mathrm{d}(\mathrm{nm})$} \\
\hline 0.3 & 15.2 & 9.4 & 7.9 & 6.3 & 5.3 \\
0.4 & 19.2 & 11.6 & 9.2 & 6.9 & \\
0.5 & 19.4 & 11.8 & 8.8 & 6.9 & \\
0.6 & 21.6 & 13.1 & 9.8 & 7.8 & \\
0.7 & 23.3 & 14.2 & 9.1 & 6.3 & \\
0.8 & 19.6 & 11.4 & 9.8 & 7.4 & 6.5 \\
0.9 & 21.8 & 12.6 & 9.5 & 8.1 & 7.3 \\
1.0 & 30.2 & 17.4 & 11.8 & & 5.8 \\
1.15 & & 14.6 & & 7.0 & \\
1.33 & 26.7 & 11.4 & & 5.7 & \\
1.4 & 20.2 & 9.5 & & 4.8 & \\
1.5 & 16.8 & 8.7 & & & \\
1.6 & 16.1 & 8.4 & & & \\
1.7 & 14.5 & 7.7 & & 6.0 & \\
1.8 & 13.6 & 6.5 & & 6.0 & \\
1.9 & 13.4 & 6.5 & & & \\
2.0 & 12.5 & 6.0 & & & \\
2.1 & 13.1 & 6.0 & & & \\
2.2 & 12.8 & 5.8 & & & \\
\hline
\end{tabular}

